\title{
In vivo anti-melanoma efficacy of allo-restricted CTLs specific for melanoma expanded by artificial antigen-presenting cells
}

\author{
Xiao-ling Lu $\cdot$ Xiao-bing Jiang $\cdot$ Ru-en Liu \\ Sheng-min Zhang $\cdot$ Zhi-hui Liang
}

Published online: 17 October 2008

(C) Springer-Verlag 2008

\section{Erratum to: Cancer Immunol Immunother DOI 10.1007/s00262-008-0573-7}

The corresponding author of the paper titled "In vivo anti-melanoma efficacy of allo-restricted CTLs specific for melanoma expanded by artificial antigen-presenting cells" has been corrected. The new authors and addresses are listed as below:

\section{X.-1. Lu $\cdot$ S.-m. Zhang}

Department of Biomedical Engineering, College of Life Science and Technology, Huazhong University of Science and Technology, 430074 Wuhan, China

The online version of the original article can be found under doi:10.1007/s00262-008-0573-7.

\section{X.-1. Lu $\cdot$ S.-m. Zhang}

Department of Biomedical Engineering,

College of Life Science and Technology,

Huazhong University of Science and Technology,

430074 Wuhan, China

\section{X.-b. Jiang}

Department of Neurosurgery,

Union Hospital, Tongji Medical College,

Huazhong University of Science and Technology,

430022 Wuhan, China

\section{R.-e. Liu}

Department of Neurosurgery,

China-Japan Friendship Hospital,

100029 Beijing, China

\section{Z.-h. Liang $(\bowtie)$}

Department of Immunology, Tongji Medical College,

Huazhong University of Science and Technology,

13 Hangkong Rd., 430030 Wuhan, China

e-mail: zhihuiliang67@163.com
X.-b. Jiang

Department of Neurosurgery, Union Hospital, Tongji Medical College, Huazhong University of Science and Technology, 430022 Wuhan, China

R.-e. Liu

Department of Neurosurgery, China-Japan Friendship Hospital, 100029 Beijing, China

Z.-h. Liang ( $\square)$

Department of Immunology, Tongji Medical College, Huazhong University of Science and Technology, 13 Hangkong Rd., 430030 Wuhan, China e-mail: zhihuiliang67@163.com

Tel.: +86-27-83692640 